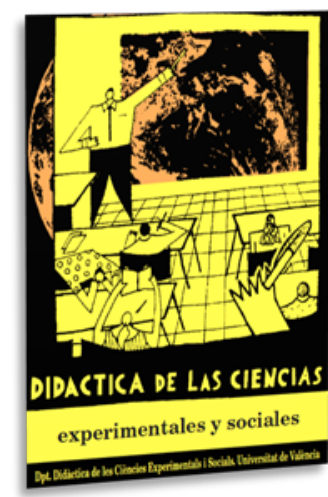

\title{
Enseñar historia en las escuelas públicas del siglo XXI. ¿Qué historia enseñar y para qué?
}

\author{
Teaching history in 21 st century public \\ schools. What history to teach and for what \\ reason?
}

DOI: $10.7203 / D C E S .40 .16835$

\author{
Gonzalo Andrés García Fernández \\ Universidad de Alcalá, gonzaloandres.garcia@edu.uah.es
}

RESUMEN: En el presente artículo tenemos como principal objetivo discutir, grosso modo, acerca de los principales debates en torno a la enseñanza de la Historia en el ámbito escolar en el siglo XXI. Si bien existe un intenso debate al respecto, también existen consensos, y nuestra labor será identificar qué preguntas han hallado respuesta, cuáles aún no y en qué ámbitos. También nos adentraremos en un análisis sobre cuáles son los objetivos de dichos debates, consensos y preguntas: si estas se enfrentan más a la forma o al fondo de la enseñanza escolar de la Historia.

Palabras Clave: enseñanza de la Historia, positivismo historiográfico, formación ciudadana, educación, pensamiento crítico

ABSTRACT: In this article, the main objective is to discuss, roughly, about the main debates around the teaching of History in the field of education in the 21st century. Although there is intense debate on this matter, there is also consensus, and our task will be to identify which questions have been answered, which have not yet, and in which areas. We will also delve into an analysis of the objectives of these debates, consensus and questions: whether they are more confronted with the way or the content of History teaching in school.

KEYWORDS: History teaching, historiographical positivism, citizen training, education, critical thinking

Fecha de recepción: marzo de 2020 Fecha de aceptación: agosto de 2020

Este artículo es fruto de los análisis y conclusiones extraídos de la tesis doctoral: García Fernández, G. A. (2019). Historia, educación y formación ciudadana, 1980-2017. Un estudio comparado en escuelas públicas de Alcalá de Henares (España) y Viña del Mar (Chile) (Tesis doctoral). Universidad de Alcalá, Alcalá de Henares. 


\section{INTRODUCCIÓN}

En este artículo nuestra principal misión será reflexionar teóricamente acerca de los principales debates que se han realizado en la temática de la enseñanza escolar de la Historia en el siglo XXI. Y sobre todo incidiendo en el carácter academicista de esta cuestión, abundando más el enfoque multidisciplinar que el interdisciplinar. Para abordar esta cuestión partimos brevemente desde la teoría de la Historia, ya que las principales líneas historiográficas desde finales del siglo XIX y principios del siglo XX (positivismo historiográfico; historicismo) siguen siendo hegemónicas en la actualidad (García Fernández, 2019: 436-442). Y esto será especialmente valioso, ya que si abordamos la enseñanza escolar de la Historia lo hacemos, pues, desde un prisma historiográfico igualmente hegemónico que denominaremos como "oficial” o "estatal”. Esta última idea será fundamental, la de la "Historia oficial", un concepto que lo definiremos como aquella selección de contenidos (hechos históricos) "sobre el pasado" (en singular) depositados bajo un relato unilineal, lógico y cuyo protagonista siempre será el mismo: la nación. De esta manera, pues, la asignatura escolar de Historia es configurada por los Estados a través de sus respectivos Ministerios de Educación con una clara finalidad educacional formativa: crear ciudadanía afín a los proyectos políticos y económicos vigentes. No se trata de una eventualidad, ya que todo ello tiene su explicación y origen en la construcción de los Estados-nación (siglo XIX); y con la necesidad de elaborar grandes relatos (Historias Generales de la nación; Historias universales) para justificar un nuevo orden político, social, económico y cultural sujeto a una estructura política denominada "Estado" y a un constructo social y cultural denominado "nación”. El pretexto homogeneizador será la formación de una ciudadanía fiel al Estado y a la nación. Para llegar a este objetivo sería necesario grandes relatos sobre "un pasado", pero también proyectados hacia un futuro (utopía liberal).

La selección de contenidos sobre "el pasado" suele articularse en dos grandes ideas. Por un lado, situamos a la Historia universal, que agrupa contenidos que tratan desde las grandes transformaciones de la modernidad del siglo XIX (revoluciones políticas, científicas, industriales) hasta los grandes conflictos de la primera mitad del siglo XX (Guerras Mundiales, Guerra fría) y los grandes acuerdos que se desarrollan durante la segunda mitad del siglo XX (ONU, Unión Europea, OTAN, fin de la Guerra Fría, etc.). Por otro lado, destacaremos el contenido de la Historia nacional, que se compondrá de un gran relato nacional diacrónico. Dicho relato vendrá a describir y explicarnos "el pasado de la nación” a través de una selección específica de hechos (políticos, bélicos, económicos, sociales $\mathrm{y}$, en último término, culturales) ordenados cronológicamente (periodos, etapas, edades) hasta el presente. Tanto la primera versión de la Historia como la segunda presentan un gran debate sobre qué enseñar y no tanto en cómo hacerlo. Todo dependerá, pues, si el/la que analiza esta discusión epistemológica y deontológica en torno a la enseñanza escolar de la Historia está de acuerdo o no con lo que se está enseñando. Y si este, de estar en desacuerdo con lo que se enseña, está dispuesto a cuestionar o no al protagonista de la Historia enseñada: la nación (Historia nacional) y el eurocentrismo (Historia universal).

\section{ENSEÑANZA ESCOLAR DE LA Historia EN EL SIGLO XXI. ¿TodAVÍA UNA HERRAMIENTA PARA CONSTRUIR CIUDADANÍA?}

Al comienzo del siglo XXI la enseñanza de la Historia seguirá siendo una preocupación tanto para los Estados como para la academia, ya que forma parte esencial de la conformación de ideas y percepciones sobre lo que sucede y ha sucedido tanto a un nivel "mundial" como nacional. Es bien conocido que, desde el siglo XIX en adelante, el interés de los Estados-nación fue, entre otras cosas, fomentar una educación cuyo objetivo fuera la configuración de una sociedad nacional. Sabemos también que los historiadores en dicha labor han jugado y juegan un rol importante. Si bien podríamos decir que la tarea (educativa) de configuración de sociedades nacionales se ha superado 
en la actualidad, la Historia (escolar) ha quedado suspendida entre los que pretenden actualizarla (revisionismos), reformarla (integrar/quitar nuevos/viejos contenidos) o, directamente, transformarla (nueva disposición deontológica-epistemológica).

Como bien sabemos los historiadores, han existido diversas formas de conceptualizar la Historia, así como el tratamiento del "pasado histórico" (debates historiográficos; teoría y filosofía de la Historia). Dichos debates han marcado tanto el quehacer historiográfico (deontológico) como el significado del propio producto historiográfico, es decir, de cómo se construye y se debe determinar el saber histórico (epistemología). Sumado a ello podemos señalar además la caída de las “viejas” formas de hacer historiografía como el romanticismo o la Historia Whig. Y aunque esto sea un hecho en la actualidad, nos daremos cuenta de que lo que subyacía en dichas corrientes historiográficas (narratividad; exaltación de los héroes e hitos políticos) en realidad no ha desaparecido totalmente. Pero ¿A qué nos referimos exactamente con todo esto? La Historia, como rama de conocimientos en el cual se alberga (supuestamente) el pensamiento y el conocimiento sobre él o lo pasado, sigue, en gran medida, ligada a los estatutos y ordenamiento del poder político. Un ámbito que habitualmente no conecta ni se relaciona con los debates y refundaciones del pensamiento historiográfico acaecido desde los años sesenta del siglo XX hasta nuestros días. El nexo establecido entre el poder político y la Historia han sido, desde el siglo XIX, e incluso en tiempos más pretéritos, una relación frecuente $\mathrm{y}$, en cierta medida, simbiótica (Benejam, Pagès Blanch, Comes y Quinquer, 1997: 16-19).

Desde finales del siglo XX y principios del XXI la preocupación por fin tradicional y escolar de la Historia, es decir, la construcción de una ciudadanía nacional se ha puesto en tela de juicio. Desde el poder (político) podríamos decir que se ha optado por una vía reformista en cuanto a "viejas" conceptualidades históricas, las visiones revisionistas de la Historia (incorporación de datos novedosos y sorprendentes), o bien por el sutil cambio de pasar de infundir "amor por la nación” al aprendizaje de saberes útiles en torno a la idea de la construcción una ciudadanía participativa (voto), responsable (derechos y deberes), tolerante (a un nivel social y cultural) y respetuosa del marco legislativo vigente. Y mencionamos que sería "sutil" ya que todo ello queda estructurado bajo las tradicionales Historias nacionales y universales que llevan funcionando desde finales del siglo XIX hasta nuestros días (Carretero, Castorina, Sarti, Van Alphen y Barreiro, 2013: 14).

De esta manera se pasa de una enseñanza de una Historia expresamente nacionalista a una enseñanza de la Historia donde se abre el abanico de contenidos, incorporando cuestiones sociales y culturales y no únicamente una "Historia del Estado y de personajes ilustres de la nación" (Carretero y Voss, 2004: 14-15). En palabras del historiador Carlos Martínez-Shaw diríamos que la Historia que se conjura como heredera del positivismo desde finales del siglo XX en adelante, tiene en vigor tres pilares fundamentales: la Historia total de los Annales, la Historia económica y, por último, el materialismo histórico de los teóricos de la Historia (Carretero y Voss, 2004: 25). Estos tres ejes configurarían el actual perfil de la Historia fruto de la superación del positivismo clásico, caracterizada por un enfoque más multidisciplinar que abarcaría una Historia más "completa" (política, economía y sociedad). Al mismo tiempo que esto sucede, la enseñanza de la Historia escolar seguiría rigiéndose bajo los márgenes del positivismo clásico, concentrándose en una Historia político-institucional de las naciones cuya metodología involucraría un aprendizaje pasivomemorístico que incidiría en un ejercicio de simplificación de los sucesos históricos (reduccionismo) (Carretero y Voss, 2004: 37-38). A pesar de la existencia de esta "nueva Historia”, ésta no se libraría de herencias positivistas como su relación con el pasado (concluso), su búsqueda por contar la verdad en la Historia y su fin objetivista (tratamiento científico de la Historia). A su vez, tampoco llegará a permear completamente en las instituciones educacionales, las cuales siguen ensimismadas en una enseñanza de Historias nacional-positivista, aunque con un perfil del siglo XXI, el cual insiste en una enseñanza de la Historia dentro del marco del civismo, la 
responsabilidad social y la diversidad cultural, racial, sexual y de género (Carretero y Voss, 2004: 48).

Ahora bien, la enseñanza escolar de la Historia en la actualidad implicaría una incómoda armonía entre el ideal romántico e ilustrado en la Historia, siendo objetivo del primero la construcción nacional-identitaria, mientras que el segundo ideal (también denominado como "disciplinario") buscaría en el estudiante la incorporación de conocimientos sobre el pasado histórico de la correspondiente nación. Es incomoda ya que el ideal romántico choca irremediablemente con el ideal ilustrado ya que este puede encontrarse con elementos del pasado que pueden interferir en la construcción de identidades nacionales, como es, por ejemplo, el propio entendimiento de que la nación es una construcción del Estado y no al revés (Carretero, et al., 2013: 14-15). Estas interferencias en la enseñanza de la Historia en las escuelas actuales tendrían su principal explicación en la irremediable misión del Estado por ligar a sociedades diversas y plurales (principal emblema de la sociedad del siglo XXI) a un elemento homogeneizante como lo es la nación (principal emblema de la sociedad del siglo XIX) a través de Historias que explican "la verdad histórica” de una nación en un eje cronológico de progreso construido todavía en términos de modernidad y objetivismo. Esto provocaría una percepción generalizada de la Historia nacional como parte de un relato verdadero, monolítico y objetivo lo cual dificulta cualquier tipo de pretensión desde la Historia por construir ciudadanos realmente críticos (Carretero, et al., 2013: 16).

La intencionalidad por reformar y no transformar la enseñanza escolar de la Historia es una cuestión que, como hemos mencionado anteriormente, la podemos ver a partir de finales del siglo XX. Según Pluckrose, la Historia escolar deberá desde la niñez, por ejemplo, “desarrollar un interés por el pasado", "comprender los valores de nuestra sociedad”, "distinguir entre los hechos históricos y su interpretación”, “demostrar que conocen algunos sucesos relevantes y pintorescos del pasado", "hacer reconstrucciones imaginarias de situaciones pasadas que estén de acuerdo con los datos disponibles" o "demostrar qué saben de algunos sucesos esenciales de la historia británica y mundial en un contexto cronológico amplio” (Pluckrose, 2002: 23-25). Los objetivos de enseñanza de la Historia escolar en la Gran Bretaña de los años noventa es un caso ejemplarizante que nos ofrece Henry Pluckrose, dándonos a entender cómo la Historia nuevamente se enfrentará a su histórico y a la vez reciente objetivo de crear ciudadanos críticos, pero a la vez leales a la nación.

Autores como Mario Carretero han trabajado, junto con otros investigadores, y en el marco de la didáctica de la Historia y la enseñanza escolar de la Historia en la actualidad. Uno de los propósitos de dichas investigaciones fue conocer la situación de esta con respecto a los fines curriculares de la misma: ¿la Historia para formación de una ciudadanía nacional o globalizada? Dicha interrogante se llevó, pues, a un punto intermedio (ni lo uno ni lo otro), lo cual conllevó a la problematización sobre los usos de la Historia en la escuela, pero también de las limitaciones del docente de Historia. Las propuestas para solucionar dicha encrucijada en torno a la enseñanza de la Historia escolar reciente coincidieron en ofrecer, pues, conocimientos históricos de carácter holístico, más convenientes y orientados hacia una mejor comprensión del mundo en el que vivimos (Carretero y Castorina, 2010: 10-11). Pendiente quedaron las herramientas de pensamiento y crítica que ayuden a sensibilizar a los estudiantes a relacionarse (mejor) epistémicamente con un mundo en constante cambio. Evidentemente esta última idea requeriría, por ejemplo, de un desmantelamiento de las disciplinas (ciencias sociales) monolíticas tal y como las conocemos en la actualidad, para avanzar hacia una nueva conceptualización de la educación. Es decir, pasar del "educar en conocimientos” al educar en herramientas para la generación de pensamiento autónomo, libre y crítico.

Al mismo tiempo que se cuestiona críticamente todo lo que involucra el destino de la Historia en la educación, no se lograría conectar esta problemática con estudios interdisciplinares que den “voz” a los estudiantes (etnografías) (Pagès y Plá, 2014: 33) y que, al mismo tiempo, se tenga en cuenta el debate sobre la enseñanza escolar de la Historia. Y no solo desde una óptica disciplinaria, sino entendida también como parte de un proyecto político que supere el marco nacional (debates 
disciplinarios y políticos al mismo tiempo). Entonces, lo que nos suscita todo esto es si efectivamente el cuestionamiento en torno al debate entre historiadores es realmente fructífero en términos prácticos (praxis), cuando realmente lo que es influyente en el aula (como veremos más adelante) es más la forma de enseñar Historia y no tanto el fondo (contenidos). De esta forma se dejaría aparcada una y otra vez la (gran) pregunta que implica necesariamente una cuestión ideológica, que nos exigiría pensar en el futuro: ¿qué sociedad queremos construir? Si no tenemos muy claro esta cuestión es un tanto complejo resolver la tipología específica de Historia que necesitamos como alternativa hoy en día. Es por ello por lo que es habitual en la actualidad que abunde una crítica hacia la Historia nacional, pero sin eliminarla del sistema educacional, entendiéndola, insistimos, desde un reformismo que sigue ligado a sus principios fundacionales de finales del siglo XIX (Carretero y Castorina, 2010: 20).

En el debate de la enseñanza de la Historia del siglo XXI interviene un tema disciplinario, pero también educacional y político. Las posturas de Mario Carretero y Marcelo Borrelli apuntan precisamente a la problemática de la Historia en las aulas, ya que el rol de la escuela queda en medio de un debate de posturas en torno a los roles de la Historia, si esta debe centrarse más en una Historia al uso (memorística, institucional-política), más crítica (incidiendo en temáticas polémicas y de gran contenido social) o en una visión actualizada de la magistrae vitae de la Historia, la cual se encargaría de mostrar un pasado histórico atroz con el objetivo de "aprender de los errores del pasado" (memoria histórica como herramienta de aprendizaje para el presente) (Carretero y Borrelli, 2010: 103-104). En este último sentido Carretero y Borrelli destacan que el uso de la Historia para juzgar al sujeto histórico (pasado) desde el presente es una tendencia de la enseñanza de la Historia escolar del siglo XXI, cuya finalidad última estaría en crear una especie de, según los autores, "memoria global" en los estudiantes, un escenario que termina por influir en los sentimientos de estos (culpa histórica, responsabilidad histórica) (Carretero y Borrelli, 2010: 105). Estos dos autores nos ofrecen ejemplos al respecto surgidos a partir de finales del siglo XX, como el estadounidense (la National History Standard y la controversia alrededor de los temas "tabú" como las bombas atómicas en Hiroshima y Nagasaki o la Guerra de Vietnam), el ruso (sustitución de la visión negativa de la URSS por una positiva que genera "orgullo patrio" en los jóvenes estudiantes), el chino (controversia social en torno a la visión "vejatoria" de la invasión a China en diferentes periodos del pasado en los libros de texto de Historia japoneses), el español (controversia por la visión reconciliadora de la Guerra Civil española y el franquismo establecida por la transición española) y el chileno (la visión sesgada sobre la dictadura militar que la clasificaría como “inevitable”) (Carretero y Borrelli, 2010: 108-110).

Finalmente, y ante el desafío de la enseñanza de la Historia en las escuelas y su rol social (formación de la ciudadanía) algunos entenderán que se deberá potenciar la enseñanza de la Historia en torno a la memoria como "exemplum" (lección del pasado al presente), en palabras del historiador búlgaro-francés Tzvetan Todorov (Todorov, 2000: 30-31). En esa línea irán importantes autores como Mario Carretero o Joan Pagés, el cual entiende la caducidad de la Historia tradicional por el auge de una Historia que relacione "presente-pasado-futuro" cuyo objetivo se centre en una praxis educacional en la que los estudiantes puedan pensar históricamente bajo criterios de libertad y crítica, alejados de la erudición positivista (Pagès, 2011: 5-6; 13-14). Historiadores como Joaquim Prats y Juan Sisinio Pérez Garzón también defienden la tesis de que la asignatura de Historia en el siglo XXI debe enseñarse lejos de su visión tradicional-nacionalista. Sisinio subraya la idea de que el mundo ha cambiado y que son necesarios cambios en la asignatura de cara a un entendimiento de nuevas identidades y pluralidades culturales en el marco de una sociedad globalizada (Pérez Garzón, 2008: 48-55), mientras que autores como Prats defienden una visión más conservadora de la Historia entendiéndola como un conocimiento que debe regirse por principios científicoexplicativos en torno al pasado en post de una construcción de una ciudadanía "pensante” y no memorística (Prats Cuevas, 2010: 14-18). 


\section{3. ¿QUÉ HISTORIA DEBERÍAMOS ENSEÑAR Y DE QUÉ FORMA SE DEBERÍA IMPARTIR EN EL ÁMBITO ESCOLAR?}

El tratamiento de forma en torno a la enseñanza de la Historia tiene implicaciones donde interviene la labor docente que se emplea en el aula, tanto a un nivel didáctico (entendimiento de los temas tradicionales a través de explicaciones innovadoras) como técnico (herramientas y recursos tecnológicos utilizados para hacer llegar el conocimiento al estudiante). Así pues, teniendo en cuenta el cómo es problematizada la enseñanza escolar de la Historia en cuanto a forma (didáctica y técnica), nuestro objetivo en este apartado es sintetizar el debate actual en el ámbito de los historiadores/as alrededor de la enseñanza escolar de la Historia en el siglo XXI en un ámbito general (cómo problema/desafío internacional).

¿Cuáles son las principales preocupaciones de los estudios que intervienen en la forma de enseñar Historia en las aulas? Si tuviéramos que resumirlo en pocas líneas diríamos que dichas inquietudes académicas enfocan sus investigaciones principalmente: 1) en la forma que debe aprenderse los contenidos de Historia (pensamiento versus memorización); 2) en los recursos de los cuales dispone el profesor para la enseñanza de la Historia en las aulas (lenguaje, recursos digitales, infraestructuras, TIC’s, etc.); 3) en la crítica hacia la forma tradicional de transmisión de conocimientos o clase magistral (nuevos roles de los docentes, formación pedagógica del profesorado, mayor participación del estudiante, etc.); y 4) en la forma de evaluación de la Historia escolar (crítica a los tradicionales exámenes escritos, nuevas formas de evaluación, etc.).

Las cuatro vías de investigación pedagógico-didáctico de la enseñanza de la Historia escolar que hemos señalado no necesariamente están aisladas, por lo que es habitual en muchas ocasiones ver trabajos que se ocupan de más de un problema a la vez en sus investigaciones y estudios de caso.

Al mismo tiempo, los debates sobre la forma no son incompatibles con los de fondo, es decir, los que intervienen el contenido de la Historia, el "qué se enseña”. En este sentido la enseñanza escolar de la Historia en el siglo XXI se ha debatido aisladamente desde la forma (didáctica, pedagogía), asimilando que el contenido (fondo) es “correcto" o "apto" para la formación de la ciudadanía actual. Por otro lado, los estudios de innovación en la metodología pedagógica o técnica pueden a su vez criticar el contenido tradicional de la asignatura de Historia en la escuela.

Las principales líneas de debate en torno a los contenidos de la enseñanza escolar de la Historia tienen cuatro grandes líneas de investigación epistemológico-deontológica: 1) La defensa de la Historia como asignatura fundamental en la construcción ciudadana y el pensamiento crítico en los estudiantes; 2) Cuestionamiento y crítica a la Historia nacional y universal en las escuelas; 3) La defensa de la interdisciplina en la Historia en el marco de las ciencias sociales; 4) Inclusión de viejos y nuevos actores en la asignatura de Historia (mujeres, diversidad sexual, diversidad racial, diversidad étnica, etc.).

Sobre las principales investigaciones en torno a la forma de enseñar la Historia podemos destacar en primer lugar las posturas metodológicas de autores como Juan Sisinio Pérez Garzón, Sebastián Plá, Joaquim Prats, Antoni Santisteban Fernández, Joan Pagès o Mario Carretero, entre otros, quienes apoyan el desarrollo de los contenidos de Historia en el aula de forma reflexiva y no memorística, señalando también a la tradicional forma de ofrecer las clases de Historia (erudición versus pensamiento histórico/crítico) (Pérez Garzón, 2002: 20-22; Prats, 2001: 31-32; 36-37; Prats, 2010: 13-14; Pagès y Plá, 2014: 14-16; Plá, 2012: 172-173; Carretero, Rosa y González, 2006: 1921; 26; Carretero y Borelli, 2008, 207-208; Pagès. 2007: 210-211; Pagès y Santisteban, 2018: 1213; Hernández Cervantes y Pagès, 2016: 128-131; 137; Prats, 2017: 16-17). Existe una especie de consenso entre el sector de la didáctica de la Historia en torno a la idea de que la Historia, independientemente de cómo se conceptualice esta (más, menos o nada científica), tiene que ser parte de un proceso de transformación didáctica. Sobre todo, por la importancia que se les da a los contenidos de esta asignatura para la configuración de una ciudadanía, responsable, tolerante y 
consciente de lo que ha sucedido. Es por ello por lo que este consenso entre los grandes especialistas de la didáctica de la Historia defiende el pensamiento histórico y la crítica en la enseñanza, ante el tradicional sistema de educación por el cual los estudiantes deben aprender y memorizar muchos contenidos sobre "el pasado" que no llegarían a comprender y que, por lo tanto, generaría un desprecio o desapego hacia la asignatura. Este punto que implica un cambio en la forma de dar la clase de Historia también interviene, en ocasiones, el fondo (contenidos). Juan Sisinio, por ejemplo, entiende que dicho cambio debe venir de la mano de una transformación más radical de la enseñanza de la Historia que deje atrás el modelo de sociedad nacional para embarcarnos en un proyecto ciudadano "transnacional" bajo el marco de una democracia “pluralista” (Pérez Garzón, 2008: 54-55).

En añadidura a estos análisis, autoras como Margarita Limón Luque entiende que la enseñanza escolar de la Historia contribuye en la actualidad a ofrecer tres objetivos fundamentales: 1) conocer "el pasado"; 2) desarrollar una identidad social/ciudadana en los estudiantes (sentido de pertenencia); 3) desarrollar intelectual, afectiva y emocionalmente a los estudiantes ("emociones sociales”) (Limón Luque, 2008: 96). Según Limón Luque, dicha enseñanza monolítica de un pasado debería repensarse en torno a "la posibilidad de trabajar de modo específico el pensamiento epistemológico de los estudiantes y facilitar la reflexión general sobre cómo se adquiere y se construye el conocimiento" (Limón Luque, 2008: 99), al desarrollo de la identidad social desde la diversidad y la pluralidad del individuo (Limón Luque, 2008: 101-102) y a un uso de la Historia destinado a la disminución de los estereotipos socio-culturales y a caminar hacia una utilización de la empatía histórica responsable (Limón Luque, 2008: 105-106). En coherencia con lo anteriormente explicado debemos comentar que precisamente donde más se suele incidir desde la enseñanza escolar de la Historia es en cuestiones tipo técnico-pedagógicas y/o tecnológicas, argumentando cómo el método "tradicional” de enseñanza ha quedado obsoleto y es por esta razón fundamental por la cual la Historia (como asignatura escolar) "no interesa” a los jóvenes (Prats Cuevas, 2017: 18-19). En este mismo hilo conductor se argumenta que la Historia (entendida como “el pasado”) sí que interesaría a los jóvenes por lo que el fallo residiría en el cómo se transmiten estos conocimientos al estudiante. En este sentido veríamos cómo se focaliza el problema en la forma (pedagogía) y en el medio (tecnología) por el cual se hace llegar el conocimiento del pasado al estudiante.

Parece evidente que la importancia de lograr conectar los contenidos de Historia con los estudiantes escolares en el ámbito escolar es fundamental. Ante dicho desafío algunos autores señalan que dicha conexión muchas veces no se alcanza a causa de la pedagogía, pero también del manejo de conocimiento del pasado de forma monolítica y alejada del presente (Prats Cuevas, 2017: 17-19). Por otra parte, existen otros estudios que entienden que la Historia ha fracasado como asignatura relevante en los jóvenes, sobre todo por carencia de innovación en los métodos de enseñanza, aun anclados en el siglo XX. También se ha entendido este desafío-problema desde los procesos de evaluación de la Historia escolar, ya que estos suelen preocuparse más por un aprendizaje pasivo-memorístico en el estudiante que por un aprendizaje facultades, como lo son la oralidad (retórica) y/o la expresión corporal (González González, 2018: 53-55). En coherencia con esta última cuestión también se ha insistido en la formación del profesor escolar como origen del problema, ya que al llegar el profesor desde el aula universitaria al aula escolar todo se vuelve más complejo, situación que conlleva a la creación de discontinuidades y alejamientos entre profesor y estudiantado. Para comenzar a solucionar esta situación algunos piensan que, por ejemplo, el aula no debería ser el único espacio de interrelación con el pasado, sino que también lo debería ser el exterior del centro escolar (enseñar más allá del aula). Otros también señalan la problemática relación de aprendizaje existente entre el estudiantado y los tradicionales libros de texto de Historia, ya que en ellos se pueden llevar a cabo importantes y diferentes avances en la adquisición de los saberes de la asignatura (García Luque y De la Cruz, 2018: 72-77). Y ante estas propuestas existen otros estudios que apuntan más ambiciosamente a que la formación del profesorado escolar de 
Historia no debe limitarse únicamente a la innovación docente en recursos y nuevos espacios, sino que además se les debería empoderar intelectualmente. Todo ello con el objetivo de lograr un espacio docente (aula) donde se genere conocimiento a través de las propias experiencias del profesorado, para así no caer en una obsolescencia pedagógica (San Pedro, López y Suárez, 2018: 60-65).

Otra de las formas que se busca desde la didáctica de las Ciencias Sociales para acercar la asignatura (Historia, pero también la Geografía y otros saberes sociales) a los estudiantes es romper la dinámica habitual entre profesor (emisor unidireccional) y estudiantado (receptores pasivos). Algunos autores entienden que dicha dinámica está obsoleta y por ello debería cambiar el formato de clase magistral por una más participativa, donde el estudiantado no se limite únicamente a participar en ciertas actividades programadas, sino que además perciba que puede participar, y que dicha acción podría llegar a ser valiosa para el resto de sus compañeros/as (Pineda-Alfonso, 2018: 80-87).

La implementación de diferentes formas narrativas en el aula como la novela en la asignatura de Historia también forma parte de la innovación en las clases, ya que puede fomentar la imaginación histórica en el estudiante, partiendo de una premisa algo discutible como lo es el afirmar que la Historia no es una narrativa (Pardo y Vidal-Abarca, 2018: 170-175). Pero no solamente utilizar la narratividad de forma pasiva, sino que también, y en la idea del impulso del pensamiento narrativo en el estudiante y el fomento de capacidades en torno a la escritura histórica ("reconstrucción del pasado") a través de la escritura creativa como forma de aprendizajecapacitación (Fernández-Rufete y Fernández-Rufete, 2018: 157-158; 160-166). Y en esta intencionalidad de incorporación de recursos e interacción entre contenidos, profesor y estudiantes también podríamos agregar la implementación del cine (narrativa audiovisual) fórum (desarrollo del pensamiento crítico oral) en la enseñanza de la Historia en aulas (González Valencia, 2015: 202205).

Por último, también debemos señalar la existencia de esfuerzos académicos colectivos tanto en general (Ciencias Sociales) como de forma específica (Historia) por acercar los conocimientos de "el pasado" a los estudiantes a través de las denominadas TIC (Tecnologías de la información y la comunicación) como medio de conexión eficaz entre contenidos (habitualmente aburridos) y estudiantes, habitualmente desconectados de contenidos que serían percibidos como poco o nada interesantes. En este sentido la informatización (recursos multimedia) no debería limitarse únicamente al aula (espacio), sino que también debería alcanzar la propia concepción de la enseñanza escolar de la Historia (Murcia, Tejedor y Lancheros, 2017: 215-217; 225; MiguelRevilla, Sánchez-Agustí y Moro-Bengoechea, 2018: 69-72; 78-79; Bernal Acevedo, 2017: 54-56). Hay trabajos que apuntan directamente al acercamiento tanto del profesor (formación técnica) y del estudiante (aprendizaje) hacia una enseñanza de la Historia “virtual”, lo cual llevaría al estudiante a una experiencia de inmersión (o simulación histórica, siendo el estudiante el protagonista del relato histórico) que no tendría con los medios tradicionales (libro de texto, clases magistrales, etc.) (Sánchez Agustí, 2004: 3-9; Egea, Arias y García, 2017: 37-38; Iturriaga Barco, 2015: 217-221; Bel Martínez y Colomer Rubio,2018: 243-249). El término que se suele utilizar para la implementación de juegos con la enseñanza es el de "gamificación”, un recurso TIC que ayudaría al docente con su trabajo y al estudiante con su formación interactiva con la asignatura de Historia (recreación histórica) (De la Calle, 2015: 75-77).

Por otra parte, las cuestiones de fondo se abordarán desde la crítica y los fines de los contenidos de Historia (unos más moderados y otros más incisivos), así como en la defensa de la (disciplina) Historia como dispositivo fundamental en la educación (formación) de la ciudadanía. Ambos elementos están insertos en un debate fundamental sobre si la enseñanza escolar de la Historia debe aceptar que el mundo está cambiando o, por el contrario, insistir en que la futura ciudadanía debe continuar rigiéndose bajo un reformismo educativo dentro del marco de las tradicionales Historias nacionales y universal-eurocéntricas. Hemos insistido también en que estas 
Historias tuvieron su razón de ser durante el siglo XIX y, de forma más importante, en el siglo XX, pero lo que realmente se está cuestionando ahora desde la didáctica de la Historia es precisamente si se debería reflexionar sobre el auge de los nuevos historicismos y positivismos en los contenidos de Historia escolar; y si se debería hacer algo al respecto ante un mundo cada vez más cambiante, móvil, complejo y diverso (Parra Monserrat, 2015: 38-39; Pérez Garzón, 2008: 40-42). Al mismo tiempo, este debate queda ligado al todavía vigente problema de los subjetivismos versus objetivismos; si efectivamente el historiador/a debe reconocer su subjetividad (interpretación de las fuentes) en post de una intersubjetividad (consensos historiográficos) o bien, optar por una suerte de autorepresión interpretativa (anular el sujeto-historiador en la obra historiográfica) con la finalidad de conseguir una objetividad en la enseñanza de un pasado monolítico (búsqueda de la verdad y reconstrucción del pasado como fin de la historiografía).

$\mathrm{Y}$ ante todo este debate (forma-fondo) nos hacemos a continuación la misma pregunta que se hizo el historiador catalán Josep Fontana en un artículo suyo del año 2006: “¿Qué Historia para el siglo XXI?”. Grosso modo, Fontana nos da a entender que la Historia que necesita el nuevo siglo no es la Historia de las guerras y los conflictos bélicos, ni tampoco de la enseñanza tradicional de lo que Fontana denomina “biografía de los Estados”, los cuales presentarían una Historia de progreso inevitable y lógico (Fontana, 2006: 5-7). Las posturas como la de Fontana, que estimulan una visión más social de la (disciplina) Historia, es decir, una Historia por y para la sociedad, y no al servicio del poder del Estado ("biografías de los Estados”), entienden que se deberían visibilizaran viejos y nuevos actores, tales como las mujeres y/o diferentes mayorías o minorías étnicas. Así pues, se parte de la constatación de que la enseñanza de la Historia tradicional ofrece un relato por y para el Estado, donde los actores son tradicionalmente hombres, blancos, heterosexuales y occidentales (Ortega Sánchez, 2018: 13-14). Dichos actores serán protagonistas de los cambios y las principales transformaciones de la nación, dejando invisibilizada a la otra mitad de la población (machismo/androcentrismo) (Miralles y Belmonte, 2004: 8-9) y a diferentes actores por su color de piel (racismo) (Rosa Ribeiro, 2013: 283-284; 295), orientación sexual (homofobia) (Tournier, 2004: 13-14; 271-275) o procedencia geográfica (eurocéntrica/xenofobia). En la actualidad, la lucha por introducir una diversidad de actores en la enseñanza de la Historia está vigente, provocando un cambio trascendental en lo que se cuenta y el impacto que este relato tiene sobre el estudiantado del siglo XXI, dando espacios a actores que normalmente no tienen espacios significativos en el relato histórico oficial. La diversidad en la enseñanza de la Historia pasa obligatoriamente por introducir a nuevos actores, pero no cayendo en la tradicional forma eurocéntrica y androcéntrica de hacerlo, sino dando voz a nuevas interpretaciones y enfoques plurales sobre los diferentes colectivos que hasta el momento han sido (y son) marginales o, sencillamente no existen, por ejemplo, en los libros de textos o en los currículos de Historia (Pagès y Sant Obiols, 2011: 131-136). La idea de una Historia que pueda conectar con “una ciudadanía global” (Pagès, Villalón y Zamorano, 2017: 163165), conectaría perfectamente con la idea de una Historia feminista (Marina Cruz, 2010: 27-30), ya que estaríamos en consonancia con la otra idea mencionada por Fontana, es decir, hacer de la Historia un saber más social y menos erudito y exclusivo.

\section{CONCLUSiOnES FinALES}

Tras revisar la gran complejidad del debate acerca de la enseñanza escolar de la Historia nos surgen algunas incógnitas al respecto ¿Cuáles son los principales motivos que impiden o entorpecen los cambios significativos (fondo y forma), tanto en los sistemas educacionales en general como en la enseñanza escolar de la Historia en particular?, ¿qué importancia adquiere la escuela y la universidad en todo esto?, y, sobre todo, ¿qué factores intervienen para que no se generen cambios estructurales en el ámbito de la enseñanza escolar de la Historia a pesar de las investigaciones y diagnósticos críticos ya realizados al respecto? Si bien no es tarea sencilla responder estas 
preguntas, expondremos a continuación de forma ordenada algunas pistas, reflexiones y potenciales vías de investigación que se sumen a todo lo que ya se ha investigado hasta el momento.

\subsection{Los motivos estructurales}

Si situamos “los grandes porqués” de este asunto sin duda nos tropezaremos una y otra vez con una razón histórica: el fenómeno histórico de la profesionalización de la (disciplina) Historia. La extensa discusión filosófico-teórica, aun inacabada, acerca del control (hegemónico) sobre qué entendemos por Historia y por su función científica y/o social será parte importante de lo que explicaremos a continuación. Sin ánimo de reproducir acá estas intensas y complejas discusiones, podremos decir que aquí está el primer motivo: el (eterno) debate deontológico y epistemológico de la (disciplina) Historia.

Mientras algunos entienden que la ciencia histórica es parte de un saber racional, teleológico y humanístico que busca, ordena y estudia verdades arrojadas en un pasado "roto", inconexo y fragmentario (historicismos), otros conciben que la (disciplina) Historia es la encargada de investigar objetivamente el pasado (singular, unilineal) a través de una metodología científica (positivismos). Pero también nos encontraremos con visiones más literarias y alejadas de los habituales formalismos en la (disciplina) Historia (romanticismos), centradas en la descripción y valoración de sucesos (hechos) históricos; también con enfoques historiográficos centrados tanto en lo mundano como los sucesos de carácter político-institucional (historiografía whig). No obstante, existirán escuelas historiográficas que entenderán que la Historia deberá abordar estudios del Unotodo en el pasado o, dicho de otra manera, que deberá establecer la investigación histórica desde varias perspectivas temáticas (más allá de lo político-institucional) y disciplinarias (Ciencias sociales) para estudiar los hechos históricos, con el objetivo teleológico de conformar "Historias totales" (Escuela de los Annales). Y ante todo esto se manifestarán los denominados "giros historiográficos" que, desde su impulso posmoderno, irrumpirán en este debate sobre el control (hegemónico) del pasado para identificar y cuestionar críticamente estas corrientes de pensamiento historiográfico.

Así pues, podríamos decir que nuestro primer motivo lo situaríamos, por una parte, en la no o poca identificación de esta cuestión teorética en la enseñanza escolar de la Historia. Por otro lado, y más importante aún, es que si llegáramos incluso a aclarar desde un primer momento este totum revolutum, veríamos que realmente los sistemas de educación no tienen este problema, ya que se moverían entre una disposición parcialmente historicista desde un punto de vista curricular y docente (el historiador-docente se limita a exponer ordenadamente "verdades del pasado" en etapas históricas conclusas), así como en una disposición parcialmente positivista a la hora de poner en marcha el aprendizaje del estudiantado (se estudia "un pasado" como parte de un proceso científico y objetivo) y viceversa (dependerá de cada caso) (García Fernández, 2019: 701-706).

Es por ello por lo que deberíamos poner en cuestión esta situación y reflexionar en consecuencia el (nuevo) rol del historiador-docente, partiendo de lo ya cuestionado (giros historiográficos), pero añadiendo una renovada disposición proactiva sobre lo que queremos enseñar y con qué objetivos. En este sentido la pervivencia de los currículos (ordenamiento de determinados hechos históricos como "la realidad histórica") van en la dirección contraria con respecto a los propósitos anteriormente mencionados. En consecuencia, deberíamos dotar de mayor importancia al historiador-docente, así como de mayor relevancia a la pluralidad y diversidad existentes en cada realidad (aula) escolar. Quizás sea aquí donde el historiador-docente deba dar un paso hacia adelante para que sea este, y no el currículo, quien decida sobre qué contenidos se deben enseñar o no en función del estudiantado. Entonces ¿Enseñar diferentes hechos históricos? Creo que la cuestión a tratar aquí no es eso. Es por este motivo por el cual debemos escapar de la idea docente de que la Historia tiene el fin teleológico de enseñar unos determinados hechos o “realidades históricas”. Quizás el rol del historiador-docente sea otro, el de dotar de herramientas 
cognitivas y críticas al estudiantado para generar pensamiento autónomo. Si para ello debemos recurrir a ciertos hechos históricos, sea pues, pero que esto no se convierta en el fin-final del historiador-docente.

\subsection{El rol de la universidad y la escuela}

Teniendo en cuenta todo lo dicho, el rol de estos dos componentes tendrá, pues, una importancia elemental. Tanto la universidad como la escuela se han encargado de dar e insuflar vida al sujeto "nación" (la que fuere). Algunos autores indican que la nación debería ser estudiada críticamente (Sáiz y López 2016: 119-125) y que, en consecuencia, huya de sus principios esencialistas, es decir, estudiar el fenómeno político e identitario que rodea al concepto nación, así como lo que conlleva la exaltación de esta (nacionalismo). Sea de una u otra manera, el (gran) problema que identificamos no está aquí realmente, sino más bien en el hecho de que la nación no ha dejado de ser el gran protagonista en los procesos de formación de historiadores-docentes y estudiantes escolares. ¿Es que no podemos dejar a un lado a la nación para emanciparnos de una vez por todas de nuestro principio fundacional-profesional? Y con esto no decimos que no deba estudiarse, pero no caigamos en el error, casi tautológico, de utilizarla como principal foco de todos nuestros análisis e investigaciones. Para ello es fundamental también despojarnos de las caducas conceptualizaciones de modernidad y progreso que impregnan la enseñanza occidentalocéntrica de las cosas. Existen más formas de ver el mundo (cosmovisiones), y es por esto por lo que deberíamos no solo integrar nuevas perspectivas, sino también preocuparnos por empoderar al estudiantado en capacidades críticas, imaginativas y proactivas para que, en última instancia, puedan dar lugar a nuevos debates y formas de entender el mundo. La universidad y la escuela han quedado atrapadas en los siglos XIX y XX. Y el siglo XXI ha quedado retratado como un auténtico revival de ambas épocas.

\subsection{Los factores del no-cambio}

Las trasformaciones que muchos compartimos no llegan a pesar de existir un variado abanico de estudios e investigaciones en el campo de la enseñanza escolar de la Historia. Algunos motivos ya han sido explicados, aunque entendemos que es necesario ahondar más en investigaciones sobre los efectos de esta situación y no tanto en el carácter del problema (diagnósticos). Evidentemente la opción ideal aquí sería un equilibrio imperfecto, que se caracterice por saber navegar en las aguas de las investigaciones que aborden y analicen cómo los contenidos de Historia (previa identificación de los mismos) son percibidos (observación pasiva) e influyen en la percepción de las cosas (observación participante) tanto en estudiantes escolares como universitarios de Historia, así como en el profesorado escolar novel de Historia. De esta manera podremos avanzar en el análisis de emisor (contenidos de Historia)-receptor (estudiantado; profesorado) que posibiliten identificar de mejor manera las consecuencias del ya identificado problema, así como de las potenciales soluciones en base a estudios empíricos (etnografía; trabajo de campo).

En esta línea de investigación existen algunos trabajos y estudios de caso que apuntan en esta dirección (García Fernández 2019: 1-26; Merchán 2007:45-47; Merchán y García-Pérez 2018: 128130), con el objetivo principal de problematizar interdisciplinarmente nuestro emisor (teoría y didáctica de la Historia) en función del estudio pormenorizado de nuestro receptor (etnografía educativa). Dicho esfuerzo interdisciplinario requerirá, además, de un esfuerzo comparativo, un escenario investigativo que nos empuja, al mismo tiempo, a abandonar la centralidad de nuestras naciones y así alcanzar una mayor perspectiva tanto del problema como de sus potenciales soluciones. 


\section{Referencias}

Bel Martínez, J. C., y Colomer Rubio, J. C. (2018). La construcción del pensamiento histórico en entornos digitales: creación y recreación por medio de la imaginación y la fantasía. En J. Monteagudo-Fernández, A. Escribano-Miralles y C. J. Gómez-Carrasco (Coord.), Educación histórica y competencias transversales: narrativas, TIC y competencia lingüística (pp. 241254). Murcia: Editum.

Benejam, P., Pagès Blanch, J., Comes, P., y Quinquer, D. (1997). Enseñar y aprender ciencias sociales, geografía e historia en la educación secundaria. Barcelona: Horsori.

Bernal Acevedo, D. (2017). TIC y educación. Creación de mundos narrativos transmediáticos en proceso de aprendizaje. Revista Luciérnaga-Comunicación, 9(18), 52-60. DOI: 10.33571

Carretero, M. y Voss, J. F. (2004). Aprender y pensar la historia, Buenos Aires: Amorrortu.

Carretero, M., Castorina, J. A., Sarti, M., Van Alphen, F. y Barreiro, A. (2013). La construcción del conocimiento histórico, Propuesta Educativa, 1(39), 13-23.

Carretero, M., Rosa, A. y González, M. F. (2006). Introducción. Enseñar historia en tiempos de memoria. En Carretero, M., Rosa, A. y González, M. F. (Coords.), Enseñanza de la historia y memoria colectiva (pp. 13-40). Buenos Aires: Paidós.

Carretero, M., y Borrelli, M. (2008). Memorias recientes y pasados en conflicto: ¿cómo enseñar historia reciente en la escuela? Cultura y Educación, 2(20), 201-215. DOI: $\underline{10.1174 / 113564008784490415}$

Carretero, M., y Borrelli, M. (2010). La historia reciente en la escuela: propuestas para pensar históricamente. En Carretero, M., y Borrelli (Eds.), La construcción del conocimiento histórico. Enseñanza, narración e identidades (pp. 103-130). Buenos Aires: Paidós.

Carretero, M., y Castorina, J. A. (2010). Introducción. En Carretero, M., y Borrelli (Eds.), La construcción del conocimiento histórico. Enseñanza, narración e identidades (pp. 9-27). Buenos Aires: Paidós.

De la Calle Carracedo, M. (2015). Tendencias innovadoras en la enseñanza de las ciencias sociales. Hacer visible lo invisible. En Hernández Carretero, A. M., García Ruíz, C. R. y De la Montaña Conchiña, J. L. (Eds.), Una enseñanza de las ciencias sociales para el futuro. Recursos para trabajar la invisibilidad de personas, lugares y temáticas (pp. 67-79). Cáceres: Universidad de Extremadura, Asociación Universitaria del Profesorado de Didáctica de las Ciencias Sociales.

Egea Vivancos, A., Arias Ferrer, L. y García López, A. J. (2017). Videojuegos, historia y patrimonio: primeros resultados de una investigación educativa evaluativa en educación secundaria. Revista Interuniversitaria de Investigación en Tecnología Educativa, (2), 2840. DOI: $10.6018 /$ riite/2017/283801

Fernández-Rufete Navarro, A. y Fernández-Rufete Navarro, M. (2018). Narrar la Historia a través de la escritura creativa: pensamiento narrativo en la enseñanza de la Historia. En Monteagudo-Fernández, J., Escribano-Miralles, A. y Gómez-Carrasco, C. J. (Eds.), Educación histórica y competencias transversales: narrativas, TIC y competencia lingüística (pp. 157-168), Murcia: Editum.

Fontana, J. (2006). ¿Qué Historia para el siglo XXI? Analecta: revista de humanidades, (1), 1-12.

García Fernández, G. A. (2019). Historia, educación y formación ciudadana, 1980-2017. Un estudio comparado en escuelas públicas de Alcalá de Henares (España) y Viña del Mar (Chile) (Tesis doctoral). Universidad de Alcalá, Alcalá de Henares, España.

García Luque, A. y de la Cruz Redondo, A. (2018). Formación del alumnado Universitario en educación patrimonial: de la académica a la sociedad. En López Torres, E., García Ruíz, C. R. y Sánchez Agustí, M. (Eds.), Buscando formas de enseñar: investigar para innovar en didáctica de las ciencias sociales (pp. 67-78). Valladolid: Ediciones Universidad de Valladolid. 
González González, J. M. (2018). Implementando con rúbricas la evaluación. Una propuesta desde el departamento de ciencias sociales de la Universidad de Extremadura. En López Torres, E., García Ruíz, C. R. y Sánchez Agustí, M. (Eds.), Buscando formas de enseñar: investigar para innovar en didáctica de las ciencias sociales (pp. 47-56). Valladolid: Ediciones Universidad de Valladolid.

González Valencia, J. A. (2015). Cine foro: una estrategia metodología para la enseñanza de las Ciencias Sociales en educación secundaria. En Hernández Carretero, A. M., García Ruíz, C. R. y De la Montaña Conchiña, J. L. (Eds.), Una enseñanza de las ciencias sociales para el futuro. Recursos para trabajar la invisibilidad de personas, lugares y temáticas (pp. 201206). Cáceres: Universidad de Extremadura y Asociación Universitaria del Profesorado de Didáctica de las Ciencias Sociales.

Hernández Cervantes, L. y Pagès Blanch, J. (2016). ¿Cómo enseñar historia y ciencias sociales en la educación preescolar?. Revista Mexicana de Investigación Educativa, 21, (68), 119-140.

Iturriaga Barco, D. (2015). Enseñar Historia hacienda visible lo invisible a través de los videojuegos de Historia en secundaria. En Hernández Carretero, A. M., García Ruíz, C. R. y De la Montaña Conchiña, J. L. (Eds.), Una enseñanza de las ciencias sociales para el futuro. Recursos para trabajar la invisibilidad de personas, lugares y temáticas (pp. 2017-222). Cáceres: Universidad de Extremadura y Asociación Universitaria del Profesorado de Didáctica de las Ciencias Sociales.

Limón Luque, M (2008). El fin de la historia en la enseñanza obligatoria. En Sánchez León, P. y Izquierdo Martín, Jesús (Coords.), El fin de los historiadores. Pensar históricamente en el siglo XXI (pp.87-111). Madrid: Siglo XXI.

Marina Cruz, L. (2010). La Historia en clave feminista. Revista venezolana de estudios de la mujer, 15(34), 27-42.

Merchán Iglesias, F. J. (2007). El papel de los alumnos en la clase de historia como agentes de la práctica de la enseñanza, Didáctica de las ciencias experimentales y sociales, 21, 33-51.

Merchán Iglesias, F. J. y García-Pérez, F. F. (2018). La enseñanza de la historia y la adquisición de competencias sociales y cívicas. En Miralles Martínez, P. y Gómez Carrasco C. J. (Coords.), La educación histórica ante el reto de las competencias: métodos, recursos y enfoques de enseñanza (pp. 127-138). Barcelona: Octaedro.

Miguel-Revilla, D., Sánchez-Agustí, M. y Moro-Bengoechea, J. M. (2018). Diseño y evaluación de un repositorio abierto de recursos didácticos para la enseñanza de la Historia reciente, EDUTEC, (64), 51-67. DOI: 10.21556/edutec.2018.64.981

Miralles Martínez, P. y Belmonte Espejo, P. (2004). Historiografía, historia de las mujeres y enseñanza de la historia en bachillerato. Alicante: XV Simposio Internacional de Didáctica de las Ciencias Sociales.

Murcia Castellanos, Y. C., Tejedor Estupiñan, M. L. y Lancheros Cuesta, D. Y. (2017). Impacto de una herramienta multimedial en el proceso de enseñanza-aprendizaje de la Historia en el aula. Revista de Medios y Educación, 50, 211-228. DOI: 10.12795/pixelbit.2017.i50.15

Ortega Sánchez, D. (2018). Las mujeres en la historia enseñada: resultados de un programa docente en formación inicial del profesorado. Enseñanza de las Ciencias Sociales, 17, 13-21. DOI: 10.1344/ECCSS2018.17.2

Pagès Blanch, J. (2007). La enseñanza de las ciencias sociales y la educación para la ciudadanía en España. Didáctica Geográfica, 3(9), 205-214. ISSN: 0210-492-X

Pagès Blanch, J. (2011). Enseñar historia, enseñar a construir el futuro. ¿Qué nos enseña la historia acerca de cómo intervenir en la construcción del futuro?, San Luis Potosí: International Standing Conference for the History of Education.

Pagès Blanch, J. y Plá, S. (2014). Una mirada regional a la investigación en enseñanza de la historia en América Latina. En Pagès Blanch, J. y Plá, S. (Coords.), La investigación en enseñanza de la historia en América Latina (pp. 13-38). México, D. F: Bonilla Artigas Editores. 
Pagès Blanch, J. y Sant Obiols, E. (2011). ¿Por qué las mujeres son invisibles en la enseñanza de la historia?, Historia y Memoria, 3, 129-146.

Pagès Blanch, J. y Santisteban Fernández, A. (2018). La enseñanza de la historia. Historia y Memoria, 17, 11-16 . DOI: 10.19053/20275137.n17.2018.8283

Pagés Blanch, J., Villalón Gálvez, G. y Zamorano Vargas, A. (2017). Enseñanza de la Historia y diversidad étnica: los casos chileno y español. Educação \& Realidade, 42(1), 161-182. DOI: $\underline{10.1590 / 2175-623661186}$

Pardo Máiquez, J. y Vidal-Abarca García, E. (2018). La narrativa histórica en el aula de educación secundaria. En Monteagudo-Fernández, J., Escribano-Miralles, A. y Gómez-Carrasco, C. J. (Coord.), Educación histórica y competencias transversales: narrativas, TIC y competencia lingüística (pp. 168-176). Murcia: Editum.

Parra Monserrat, D. (2015). ¿Hacia una nueva didáctica? Posibilidades y retos para la enseñanzaaprendizaje de la Historia en el siglo XXI. En J.C. Colomer Rubio, J. Esteve Martí y M. Ibáñez Domingo (Coords.), Ayer y hoy. Debates, historiografía y didáctica de la Historia (pp. 38-41). Valencia: Asociación de Historia Contemporánea, Universitat de València.

Pérez Garzón, J. S. (2002). Usos y abusos de la historia, Gerónimo de Uztariz, 17-18, 11-24.

Pérez Garzón, J. S. (2008). ¿Por qué enseñamos geografía e historia? ¿Es tarea educativa la construcción de identidades?, Historia de la educación, 27, 37-55.

Pineda-Alfonso, J. A. (2018). Las dinámicas participativas en los grupos de debate: un estudio de caso en la enseñanza de las Ciencias Sociales. En López Torres, E., García Ruíz, C. R. y Sánchez Agustí, M. (Eds.), Buscando formas de enseñar: investigar para innovar en didáctica de las ciencias sociales (pp. 79-88). Valladolid: Ediciones Universidad de Valladolid.

Plá, S. (2012). La enseñanza de la historia como objeto de investigación, Secuencia, 84, 163-184.

Pluckrose, H. (2002). Enseñanza y aprendizaje de la historia, Madrid: Morata.

Prats, J. (2001). Enseñar historia. Notas para una didáctica renovadora. Mérida: Junta de Extremadura.

Prats, J. (2010). En defensa de la Historia como materia educativa, Tejuelo, (9), 8-18.

Prats, J. (2017). Retos y dificultades para la enseñanza de la Historia. En Sanz Camañes, P., Molero García, J. y Rodríguez González, D. (Coords), La historia en el aula. Innovación docente y enseñanza de la historia en la educación secundaria (pp. 15-32). Lleida: Milenio.

Rosa Ribeiro, R. (2013). Letras negras, páginas brancas: as imagens do negro entre a historiografía e o ensino de história (Brasil, sefunda metade do século XX), ETD, 15(2), 281-299. DOI: 10.20396/etd.v15i2.1283

Sáiz Serrano, J. y López Facal, R. (2016). Narrativas nacionales históricas de estudiantes y profesorado en formación. Revista de Educación, 374, 2016, 118-141. DOI: 10.4438/1988592X-RE-2016-374-328

San Pedro Veledo, M. B., López Manrique, I. y Suárez Suárez, M. A. (2018). La metodología Research Based Learning y la didáctica de las Ciencias Sociales: descripción y resultados de un Proyecto de innovación. En E. López Torres, C. R. García Ruíz y M. Sánchez Agustí (Eds.), Buscando formas de enseñar: investigar para innovar en didáctica de las ciencias sociales (pp. 57-66). Valladolid: Ediciones Universidad de Valladolid.

Sánchez Agustí, M. (2004). Redefinir la historia que se enseña a la luz de las TIC. Un análisis sobre nuevas maneras de aprender Roma. Alicante: XV Simposio Internacional de Didáctica de las Ciencias Sociales.

Serrano, S. (2014). Enseñanza de la historia e identidad nacional: Un vínculo a historizar desde la experiencia chilena, 1850-1930, Encounters/Encuentros/Rencontres on Education, (15), 209222. DOI: 10.24908/eoe-ese-rse.v15i0.5340

Todorov, T. (2000). Los abusos de la Memoria, Barcelona: Arléa. 
Tournier, P. (2004). Los gays en la historia. Una apasionante crónica de la presencia e influencia homosexual y lesbiana a lo largo de los siglos. Barcelona: Ediciones Robinbook.

CÓMO CITAR ESTE ARTÍCULO

García Fernández, G. A. (2021). Enseñar historia en las escuelas públicas del siglo XXI. ¿Qué historia enseñar y para qué? Didáctica de las ciencias experimentales y sociales, 40, 35-50. DOI: 10.7203/DCES.40.16835 
